\section{Ependimoma celular parcialmente resecado complicado con meningoependimocoroiditis bacteriana por Pseudomonas aeruginosa e infección sistémica por citomegalovirus}

\section{Partially resected cellular Ependymoma complicated with bacterial meningoependimocoroiditis by Pseudomonas aeruginosa and systemic infection by Cytomegalovirus.}

Otero-Mendoza $\mathrm{FJ}^{1}$, Corcuera-Delgado $\mathrm{CT}^{2}$, Aguilar-Ortiz $\mathrm{MR}^{3}$, Gómez-Garza $\mathrm{G}^{4}$, Aguilar-Gómez $\mathrm{NE}^{5}$

\section{RESUMEN CLÍNICO}

Niño de 1 año 10 meses de edad, originario de Irapuato, Guanajuato, sin antecedentes de importancia para el padecimiento actual. Inició dos meses previos a su ingreso con crisis convulsivas tónico-clónicas generalizadas de 15 segundos de duración durante el sueño. Se realizó electroencefalograma que reportó actividad epileptiforme, por lo que se dio tratamiento con ácido valproico. Una semana previa al ingreso se agregó ataxia troncal impidiendo la marcha, por lo que se realizó una tomografía axial computarizada de cráneo en la que se observó un tumor en fosa posterior con densidad heterogénea y áreas de necrosis central que obliteraba el cuarto ventrículo, ocasionando efecto de masa y desplazamiento ventral del tallo cerebral. Por tal motivo, fue referido a nuestra institución.

A su ingreso se realizó tomografía axial computarizada donde se observó una tumoración heterogénea que ocupaba y expan-
${ }^{1}$ Médico Adscrito al Departamento de Infectología. ${ }^{2}$ Médico Adscrito Departamento de Patología. ${ }^{3}$ Médico Adscrito al Servicio de Oncología. ${ }^{4}$ Médico Adscrito al Servicio de Radiología. ${ }^{5}$ Residente Quinto Año Infectología.

Instituto Nacional de Pediatría, México. Recibido: 18 de mayo del 2017 Aceptado: 22 de mayo del 2017 Correspondencia Francisco Javier Otero-Mendoza droterom@yahoo.com

Este artículo debe citarse como Otero-Mendoza FJ, Corcuera-Delgado CT, Aguilar-Ortiz MR, Gómez-Garza G, Aguilar-Gómez NE. Ependimoma celular parcialmente resecado complicado con meningoependimocoroiditis bacteriana por Pseudomonas aeruginosa e infección sistémica por citomegalovirus. Acta Pediatr Mex. 2017;38(4):244-254. 
día al cuarto ventrículo, con compresión de las estructuras circundantes, y una gran dilatación ventricular con moderado edema transependimario. Dos días después hubo datos de cráneo hipertensivo, por lo que se colocó sistema de derivación ventrículo peritoneal de urgencia (Figuras 1 y 2). Un mes después se realizó craniectomía programada para resección tumoral administrándose 3 dosis de cefalotina como tratamiento antibiótico profiláctico.

En el tejido tumoral analizado se observó una neoplasia maligna de estirpe glial compuesta de células con núcleos regulares, redondos a ovalados y abundante cromatina granular; entre los núcleos era evidente un fondo gliofibrilar variablemente denso; con mucha frecuencia se reconocían pseudo-rosetas perivasculares en las que las células tumorales estaban dispuestas alrededor de los vasos, con una zona intermedia que constaba de prolongaciones ependimarias finas dirigidas hacia la pared del vaso (Figura 3). Las células neoplásicas también formaban estructuras pseudo-glandulares redondas o alargadas (rosetas, conductos) que recuerdan el conducto ependimario embrionario, con prolongaciones largas y delicadas que se extendían dentro de una luz. Con los hallazgos histopatológicos de obvia diferenciación ependimaria en las áreas hipercelulares, pero sin actividad mitótica evidente, necrosis, ni proliferación vascular y que alternaba con áreas hipocelulares en donde predominaba el fondo gliofibrilar, la neoplasia fue diagnosticada como ependimoma celular grado II de acuerdo con la clasificación la Organización Mundial de la Salud. ${ }^{1}$

Al décimo día de la craniectomía presentó endotropía, somnolencia e hipertensión, realizándose tomografía axial computarizada cerebral donde se encontró hematoma hiperdenso residual en el lecho quirúrgico y se observó nuevo incremento en la talla ventricular, por lo que pasó a quirófano para exteriorización del sistema de derivación ventrículo peritoneal por hemorragia intraventricular (Figura 4). Se agregó fiebre hasta $38.6^{\circ} \mathrm{C}$ de difícil control, por lo que se inició esquema antibiótico con ceftriaxona y vancomicina a dosis de sistema nervioso central, para cober-
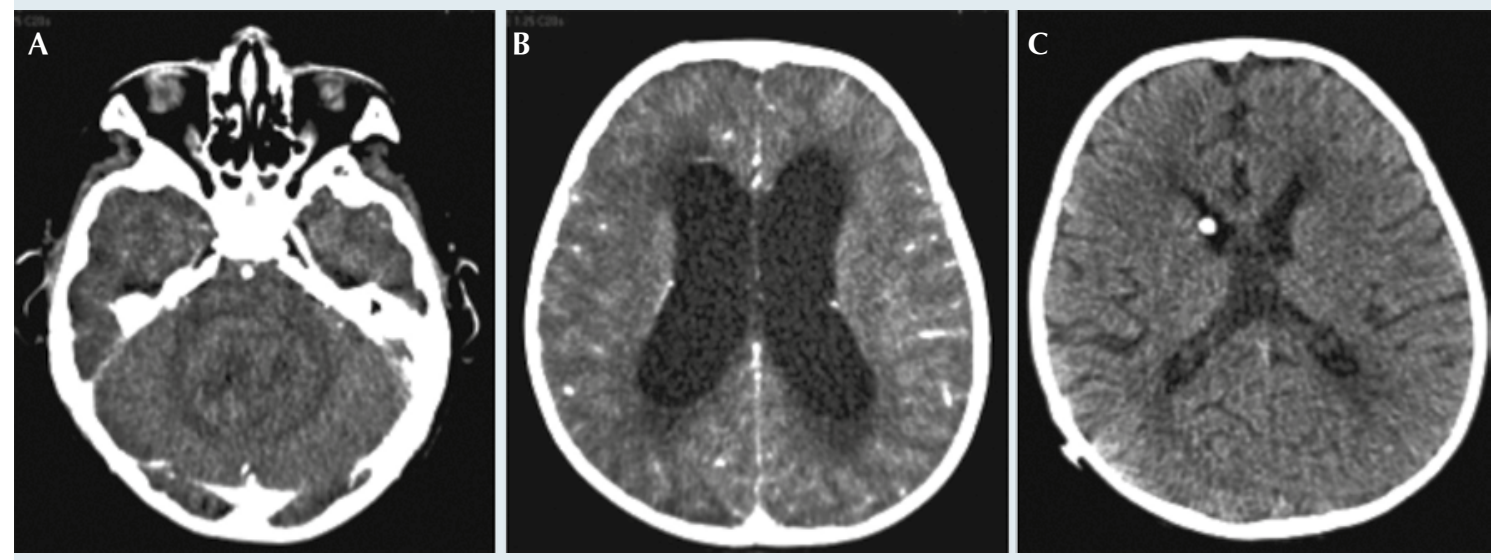

Figura 1. Cortes axiales de tomografía computada de cráneo con contraste. A) Tumor heterogéneo que ocupa y expande al cuarto ventrículo; B) Compresión de las estructuras circundantes, gran dilatación ventricular y moderado edema transependimario. C) Tomografía computada de cráneo simple post-derivación, hay disminución en la talla ventricular supratentorial y en el edema transependimario. 

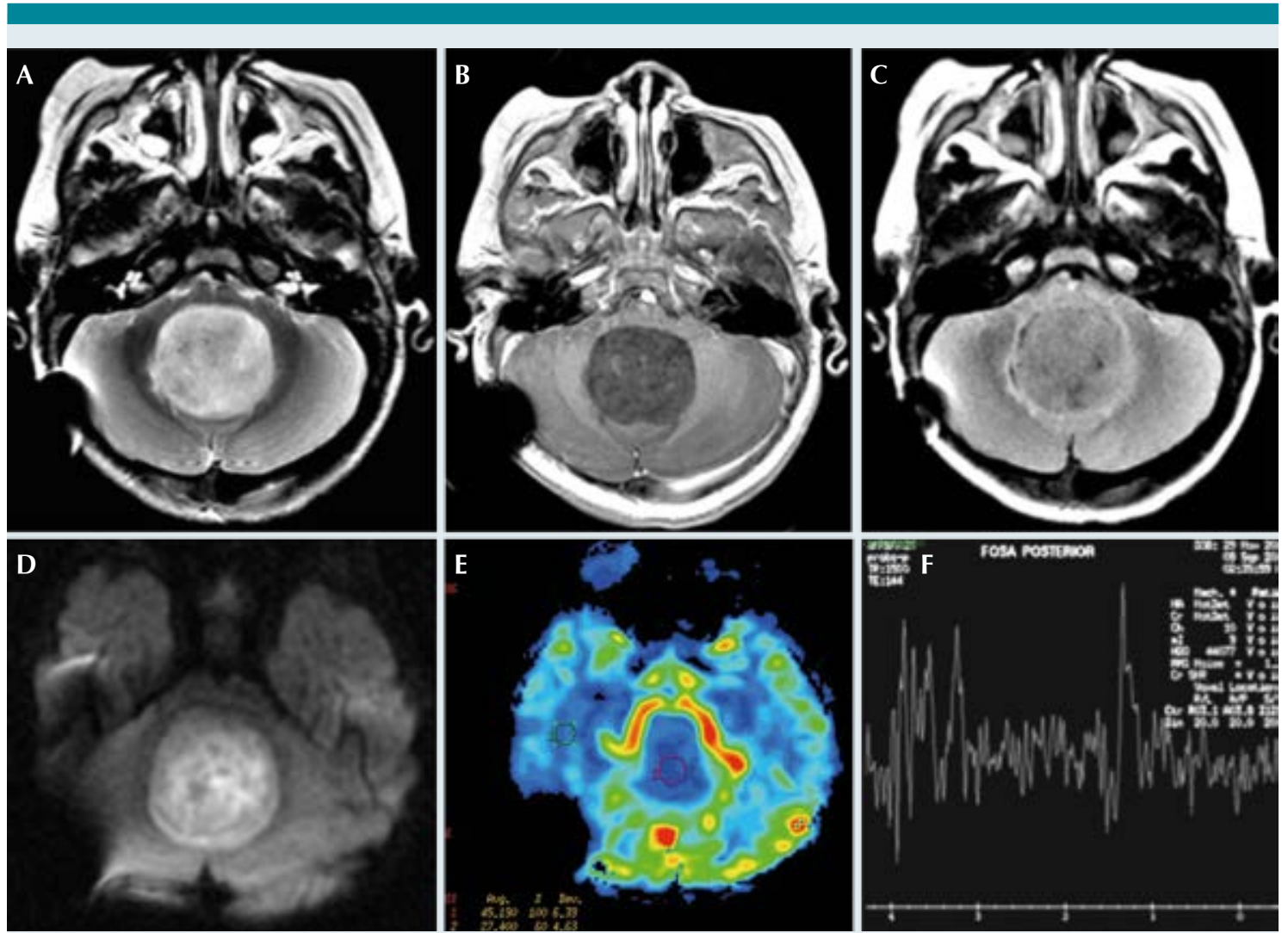

Figura 2. Resonancia magnética cerebral. A) Secuencia ponderada en T2 en el plano axial que caracteriza al tumor intraventricular con una señal heterogénea por celularidad variable. B) Secuencia en T1 post-contraste, la lesión presenta escasa vascularidad y reforzamiento. C) Imagen en FLAIR axial. Hay una adecuada interfase entre la lesión y el parénquima circundante, con escasa cantidad de edema perilesional. D-E) Mapa de volumen de perfusión cerebral relativa ( $\mathrm{rCBV}$ ) que demuestra hipoperfusión en el tejido tumoral en relación con el parénquima cerebral sano. F) Espectroscopia $\left(\mathrm{H}^{1}\right)$ con un pico alto de Colina $(\mathrm{Ch})$ con ausencia de los picos de $\mathrm{N}$-Acetil-aspartato (NA) y Creatina $(\mathrm{Cr})$; pico alto de lactato.

tura de bacilos Gram negativos y cocos Gram positivos, respectivamente. En el cultivo de líquido cefalorraquídeo hubo desarrollo de Pseudomonas aeruginosa por lo que se suspendió ceftriaxona y se inició meropenem $(120 \mathrm{mg} /$ $\mathrm{kg} / \mathrm{d})$. Se reportó antibiograma de $P$. aeruginosa con resistencia a carbapenémicos, sensibilidad intermedia a piperacilina/tazobactam y sensibilidad a cefalosporinas de cuarta generación, por lo que se suspendió meropenem y se inició cefepima $(150 \mathrm{mg} / \mathrm{kg} / \mathrm{d})$.
El paciente presentó deterioro clínico, somnolencia y dificultad respiratoria por lo que se pasó a ventilación mecánica y se realizó otra tomografía axial computarizada que mostró abundante líquido con detritus sugestivos de pus. Por este motivo, se decidió re-intervenir quirúrgicamente para retirar el sistema de derivación ventrículo peritoneal y colocar ventriculostomía externa (Figura 5). Posteriormente, continuó hemodinámicamente inestable, con datos clínicos de choque séptico sin respuesta a manejo intensivo 


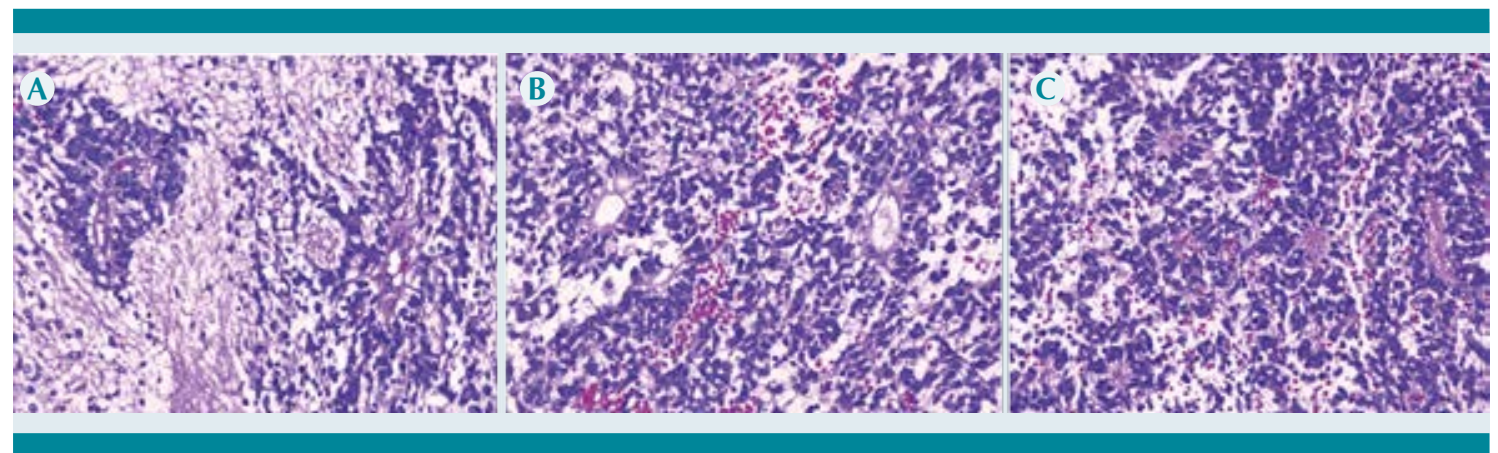

Figura 3. Biopsia del tumor cerebral. A) Pseudo-rosetas perivasculares y zonas paucicelulares con abundante malla fibrilar. B) Rosetas ependimarias en zonas hipercelulares y escasa malla fibrilar. C) Rosetas con malla fibrilar dentro de las luces en una zona hipercelular.
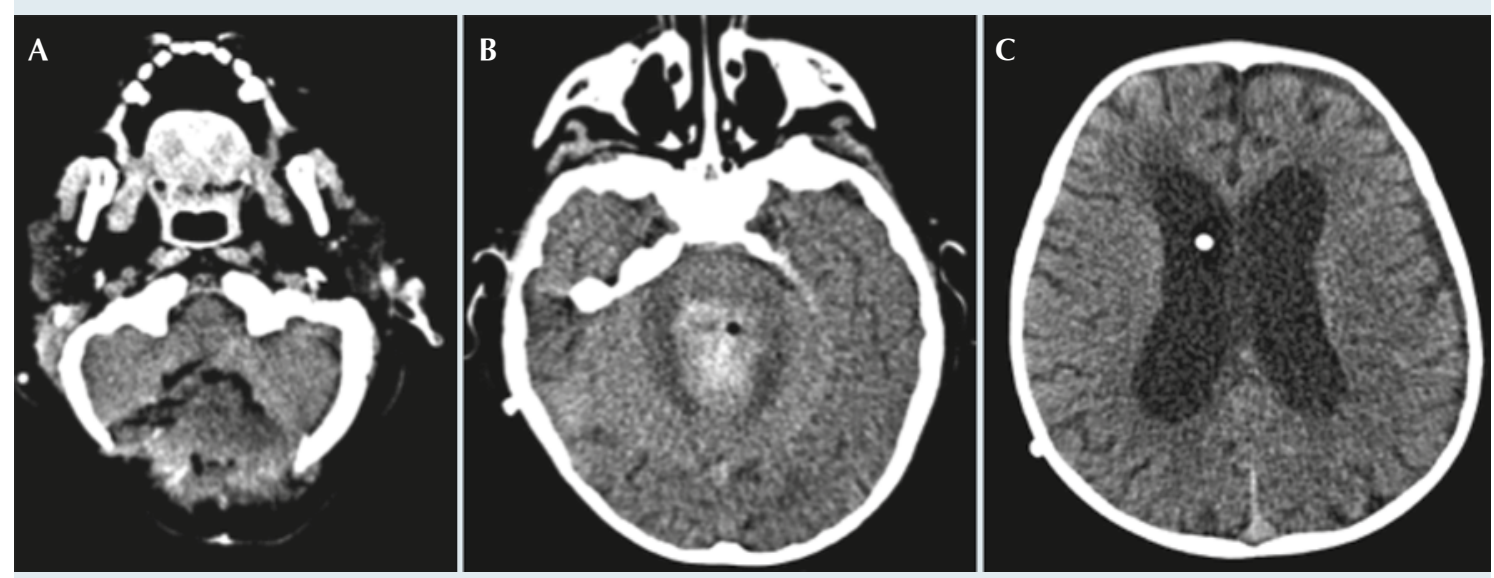

Figura 4. A) Tomografía computada post craneotomía occipital. B) Resección parcial de la tumoración intraventricular con hematoma hiperdenso residual en el lecho quirúrgico. C) Nuevo incremento en la talla ventricular.

persistiendo con taquicardia, hipertensión arterial y disminución en la saturación de oxígeno; finalmente, presentó paro cardiaco sin respuesta a maniobras de reanimación.

En la autopsia completa el encéfalo tenía zonas hemorrágicas subaracnoideas. En el corte sagital (Figura 6) se podían ver las huellas de la cirugía y la extensión de la neoplasia que dependía y destruía diferentes áreas del cerebelo y se extendía a la cisterna magna, tallo, mesencéfalo y región supratentorial; la neoplasia tenía un aspecto multinodular-arenoso mezclado con sangrado antiguo. La histopatología de la neoplasia era igual a la analizada en la resección tumoral inicial; se corroboró y diagnosticó un ependimoma residual de $3 \times 4 \mathrm{~cm}$ infratentorial que infiltraba tallo, mesencéfalo, acueducto de Silvio, tercer y cuarto ventrículo y cerebelo con extensión a la cisterna magna e implantes meníngeos cerebelosos y de la médula espinal cervical. Se observaron infartos cerebrales subagudos con microglía espumosa activada y proliferación astrocítica reactiva extensa. Además, era evidente una hidrocefalia no 

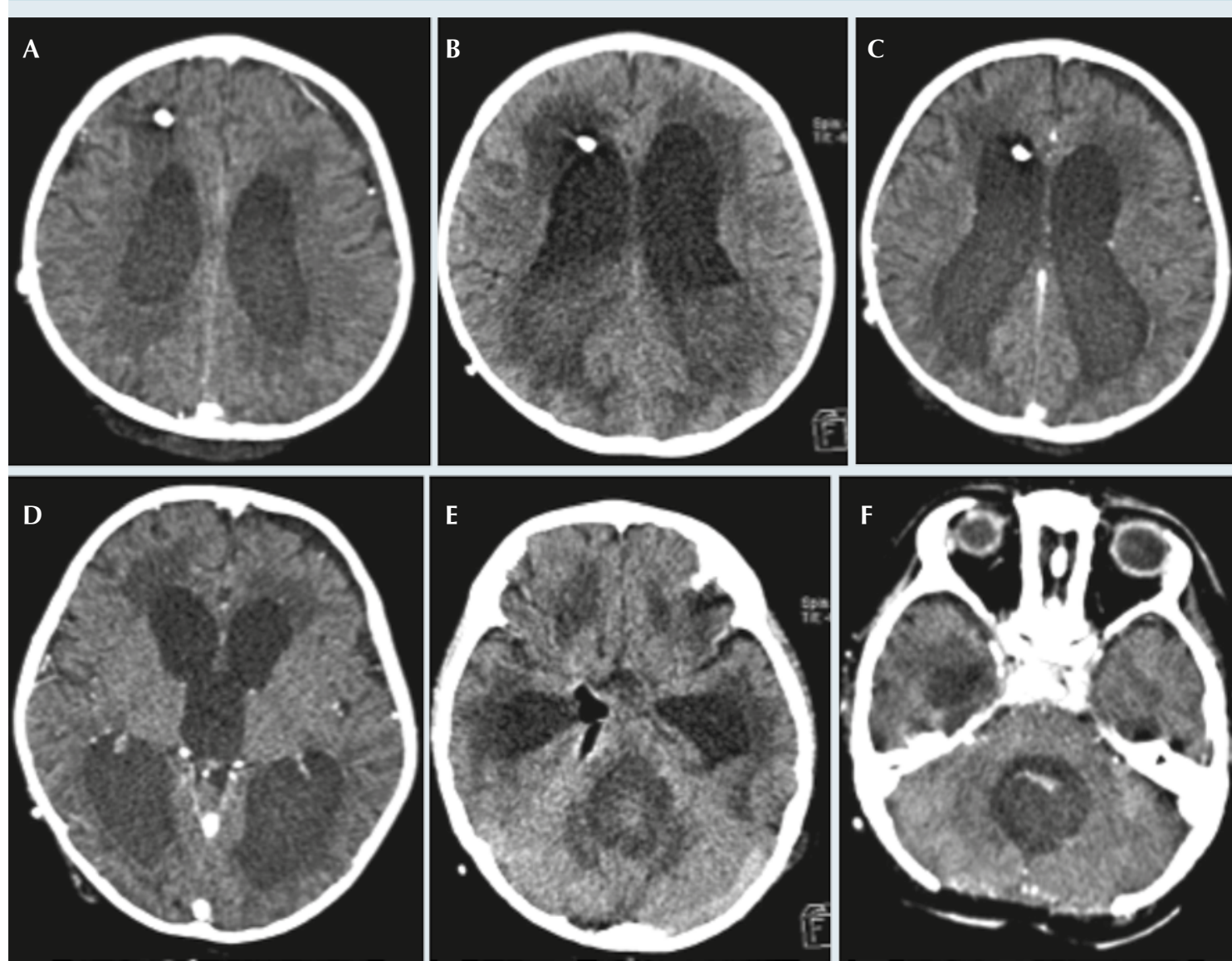

Control 10 días

Figura 5. Tomografía computada con y sin contraste de control en el día 10 post craniectomía. Se identifica una mayor dilatación ventricular con incremento en el edema transependimario, y un nivel líquido-líquido al interior de los ventrículos (flecha en B) con un material de mayor densidad en la porción más declive. Se visualiza además gas en la cisterna ambiens (flecha en E). Hay disminución en la densidad del hematoma del lecho quirúrgico en $\mathrm{F}$.

comunicante (obstructiva) secundaria. También se observó exudado en las leptomeninges sobre la superficie del encéfalo y en la ubicación de la neoplasia y lecho quirúrgico.

En la examinación histológica se observó una meningoependimocoroiditis piógena aguda (Figura 7) representada por un exudado supurativo con bacilos Gram negativos en ventrículos laterales, corroborada con el cultivo post mor- tem que reportó desarrollo de Pseudomonas aeruginosa en líquido cefalorraquídeo, pulmón, hígado y bazo. Los pulmones tenían una gran cantidad de macrófagos alveolares infectados por citomegalovirus (Figura 8). Además, se observaron cambios citopáticos virales por citomegalovirus en glándulas salivales, páncreas, suprarrenales, esófago, estómago, plexos coroides y médula espinal. Finalmente, se observaron datos anatómicos de choque: daño alveolar 


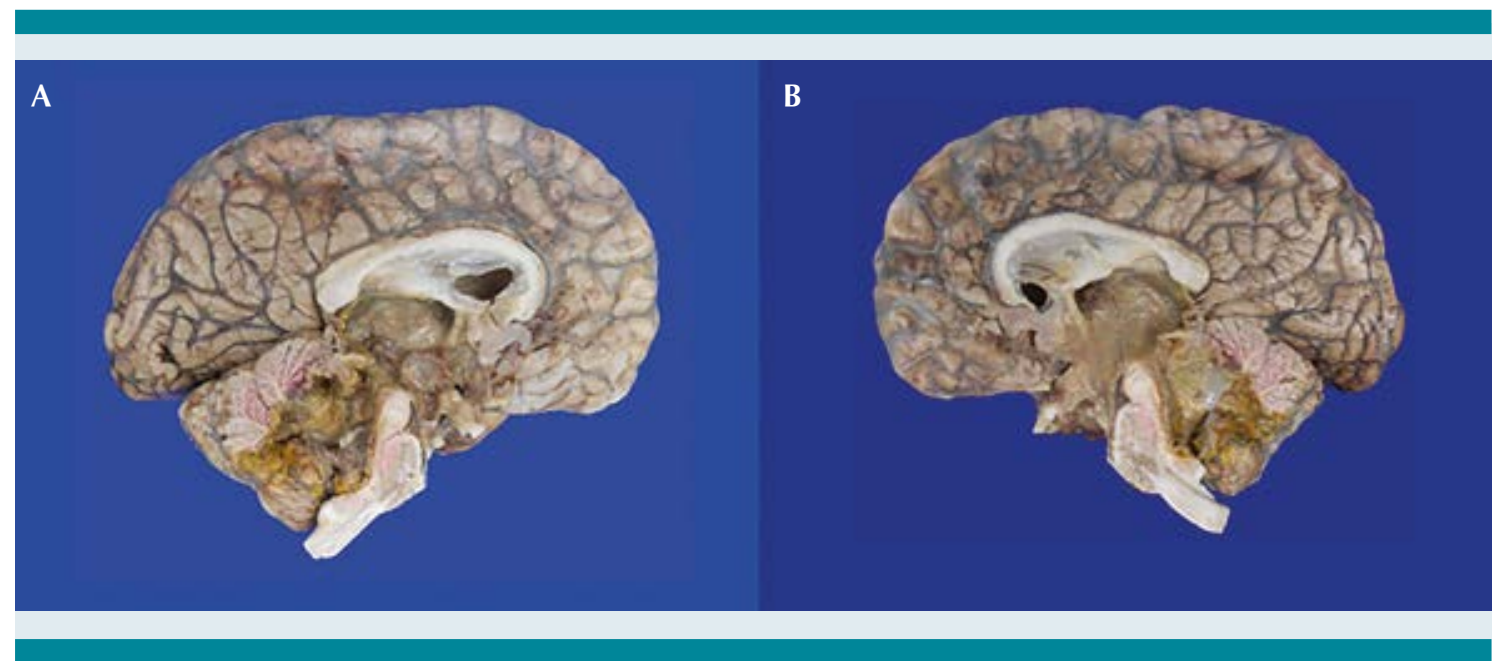

Figura 6. Encéfalo. A) Corte sagital hemisferio izquierdo. B) Corte sagital hemisferio derecho.

difuso en fase exudativa (membranas hialinas) con edema pulmonar agudo; miopatía visceral hipóxico-isquémica en corazón, tubo digestivo y vejiga; esteatosis hepática universal macro y microvesicular con colestasis leve; involución grave del timo (2 g vs. 20 g); dilatación vesical y dilataciones segmentarias del intestino delgado y colon sigmoides; nefromegalia bilateral (riñón izquierdo $65 \mathrm{~g}$ vs. $40 \mathrm{~g}$, derecho $70 \mathrm{~g}$ vs. $44 \mathrm{~g}$ ).

\section{DIAGNÓSTICOS FINALES}

\section{Enfermedad principal:}

a. Ependimoma celular grado II residual $(3 \times 4 \mathrm{~cm})$ infratentorial que infiltra taIlo, mesencéfalo, acueducto de Silvio, tercer y cuarto ventrículo, cerebelo, y se extiende a la cisterna magna con implantes meníngeos cerebelosos y de la médula espinal.

II. Complicaciones o enfermedades contribuyentes a la muerte:

a. Meningoependimocoroiditis piógena (bacteriana) aguda. b. Infección sistémica grave por citomegalovirus.

III. Causa de muerte:

a. Datos anatómicos de choque.

IV. Otros hallazgos relacionados con la atención en salud:

a. Laringotraqueítis crónica leve con metaplasia escamosa madura extensa.

\section{COMENTARIO CLÍNICO}

En general, los tumores cerebrales representan la tercera neoplasia maligna más frecuente en pediatría (15 a $20 \%$ de todos los tumores en este grupo etario), con una incidencia estimada en nuestro medio de 10.3 casos por millón por año (2015). ${ }^{2}$ Sin embargo, los datos epidemiológicos son heterogéneos; países desarrollados suelen diagnosticar y reportar más, comparados con países en vías de desarrollo; en parte por el acceso a herramientas diagnósticas (tomografía axial computarizada o resonancia magnética). ${ }^{3}$

Los ependimomas representan aproximadamente $10 \%$ de los tumores cerebrales y corresponden 


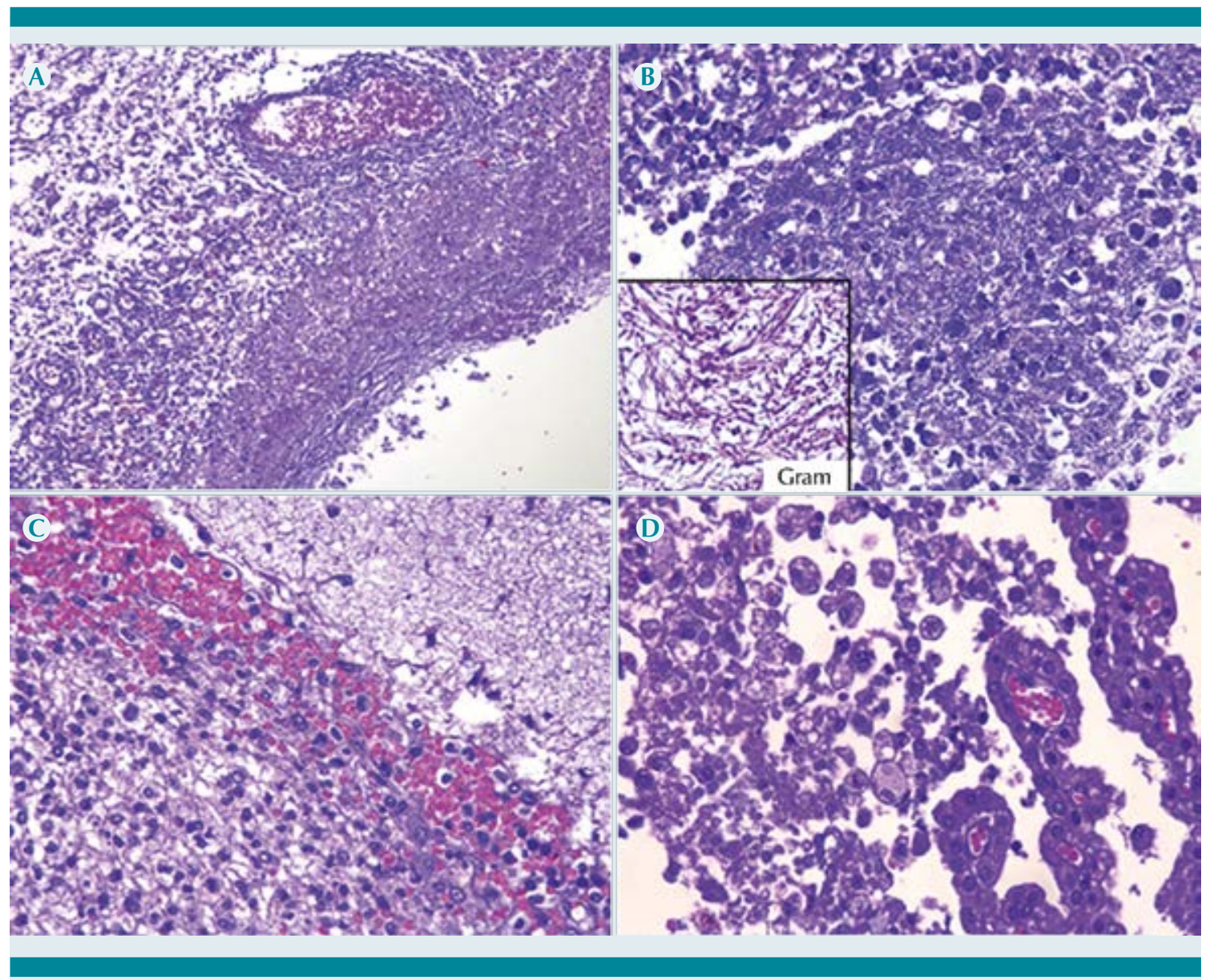

Figura 7. Meningoependimocoroiditis. A) Pared ventricular con necrosis y hemorragia antigua; B) supuración con bacterias Gram negativas; C) piamadre; D) plexos coroides. En todos se observa un proceso inflamatorio supurativo asociado con infección por Pseudomonas aeruginosa.

a la tercera causa de neoplasia maligna intracraneal. ${ }^{4}$ El tumor del paciente en discusión está bien representado en la literatura: su pico de incidencia (0-4 años de edad con incidencias reportadas de 5.2 casos por millón), su sitio de origen ( $2 / 3$ se originan en fosa posterior, sobre todo en menores de 3 años) y su localización (infratentorial, originándose en vermis o piso del cuarto ventrículo y extendiéndose al interior del ventrículo). ${ }^{4}$

La neurofibromatosis tipo 2 es el único trastorno genético asociado a predisposición para desarrollo de ependimomas (i.e. $25-70 \%$ de los ependimomas intraespinales esporádicos tienen mutaciones en la neurofibromatosis tipo 2), ${ }^{4}$ a pesar de algunas asociaciones con virus (i.e. se ha detectado genoma del virus SV40 en la mayoría de los ependimomas y carcinomas de plexos coroideos, y también en astrocitomas, meningiomas, glioblastoma multiforme y meduloblastoma); sin embargo, se han realizado grandes estudios epidemiológicos con vigilancia de hasta 30 años y no se ha detectado mayor incidencia en los individuos expuestos. ${ }^{4}$ 


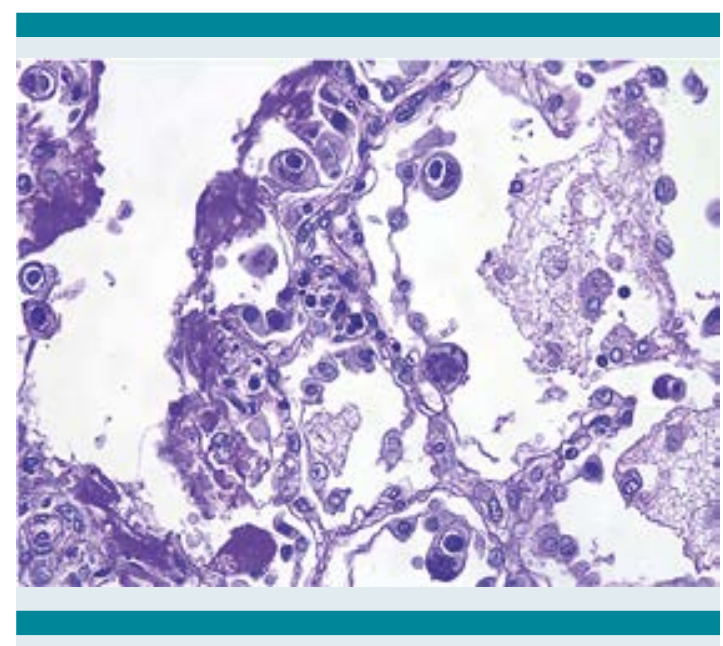

Figura 8. Citomegalovirus: inclusiones pulmonares nucleares (bien definidas) y citoplásmicas (mal definidas). Tinción con ácido peryódico de Schiff.

Radiológicamente se dice que el ependimoma es un tumor "plástico", pues puede ocupar el ventrículo y protruir a través del agujero de Luschka. También se pueden documentar calcificaciones en su interior hasta en $50 \%$ de los casos, hidrocefalia obstructiva, así como realce heterogéneo (tomografía axial computarizada/resonancia magnética). ${ }^{4}$ Clínicamente, los tumores primarios de fosa posterior se presentan con signos y síntomas de hidrocefalia obstructiva, incluyendo cefalea, vómito y ataxia. La infiltración hacia el tallo cerebral o crecimiento a través del agujero de Luschka o el canal central pueden resultar en parálisis de nervios craneales, tortícolis o meningismo. Los menores de dos años de edad tienden a presentarse con datos inespecíficos como irritabilidad, vómito, letargia, macrocefalia o incluso trastornos de la marcha, con una evolución generalmente menor a 6 meses (i.e. $50 \%$ de ellos presentan sintomatología de un mes o menos de duración). ${ }^{4}$

La resección quirúrgica completa es el factor pronóstico más importante para el desenlace. En la mayoría de los pacientes se recomienda la radioterapia concomitante como terapia adyuvante (si la edad del paciente lo permite) y se usa quimioterapia para controlar la diseminación microscópica y retrasar el inicio de radioterapia en pacientes pequeños. ${ }^{5}$ Actualmente, la supervivencia a 5 años global de los niños con ependimoma está en el rango de 50-60\%. ${ }^{5}$ La mayoría de los niños sometidos a radiación de fosa posterior para este tumor son capaces de cursar una vida escolar normal. ${ }^{5}$

Las infecciones en pacientes sometidos a craniectomía por tumor intracraneal tienen una elevada morbilidad y mortalidad, además de incrementar el tiempo de estancia hospitalaria y costos asociados con la atención médica. El diagnóstico oportuno permite el inicio de tratamiento antibiótico apropiado, así como disminuir la mortalidad. Sin el manejo antibiótico apropiado, la mortalidad en estos pacientes ha sido reportada en un rango de 15 a $70 \%{ }^{6}$ Pese al desarrollo de diversas estrategias para disminuir el riesgo de infecciones posterior a craniectomía su incidencia no ha disminuido de forma sustancial y se encuentra entre el 2 y $5 \%$, incluso $10 \%$ cuando no se utilizan antibióticos profilácticos. Además, se considera que su incidencia es mayor a la que se reporta. ${ }^{7,8} \mathrm{La}$ infección en el sitio quirúrgico se asocia con diferentes factores de riesgo como tratamiento con esteroides, radioterapia, quimioterapia, cirugía de urgencia, cirugía contaminada (intervención a través de senos paranasales o mastoides), tiempo quirúrgico mayor de 3 horas, re-intervención e infecciones previas. ${ }^{9}$

La aparición de fístulas de líquido cefalorraquídeo tras la intervención constituye otro factor que favorece este tipo de infecciones. Los procesos infecciosos pueden limitarse a piel, o bien desarrollar infecciones más profundas que pueden comprometer hueso, meninges, espacio subdural y encéfalo. ${ }^{7,10} \mathrm{El}$ tiempo entre el evento quirúrgico y el inicio de la infección varía según la publicación. Dashti y sus colaboradores reportaron un rango entre 4 días a 5 años posterior a la cirugía, con una media de 1.5 meses. $^{11}$ 
Las manifestaciones clínicas de infección son variables y dependen de la patogénesis de la infección y la virulencia del microorganismo. Los hallazgos clínicos característicos son fiebre, vómito, crisis convulsivas, cefalea y deterioro del estado de alerta (65\% de los pacientes). ${ }^{11,12}$ Además, se han reportado datos clínicos y de laboratorio de sepsis y choque séptico, así como de intubación no programada del paciente. ${ }^{11,12}$ El diagnóstico se realiza con la sospecha clínica junto con exámenes de laboratorio y gabinete. Es de vital importancia enviar líquido cefalorraquídeo para realizar examen citoquímico, citológico, tinción de Gram y cultivo. La presencia de pleocitosis, hipoglucorraquia e hiperproteinorraquia son altamente sugestivos de ventriculitis, meningitis, o ambas. La definición de ventriculitis o meningitis asociada con los cuidados de la salud de los Centers for Disease Control y de la Prevention's National Healthcare Safety Network incluye: ${ }^{12}$

a. Cultivo positivo de líquido cefalorraquídeo.

b. Al menos 2 de los siguientes signos sin otra causa aparente:

- en $>1$ año: fiebre $>38^{\circ} \mathrm{C}$ o cefalea, signos meníngeos, alteración en pares craneales, o al menos dos de los siguientes signos sin otra causa aparente:

- en $\leq 1$ año: fiebre $>38^{\circ} \mathrm{C}$ o hipotermia $<36^{\circ} \mathrm{C}$, apnea, bradicardia o irritabilidad y al menos 1 de los siguientes hallazgos de laboratorio:

- Pleocitosis, hiperproteinorraquia e hipoglucorraquia.

- Tinción de Gram positiva.

- Hemocultivo positivo.

Los estudios de imagen son de utilidad para determinar el origen de la infección (ej. extensión local de una infección adyacente) además de identificar complicaciones como hidrocefalia, vasculitis, hemorragia o trombosis venosa. ${ }^{11-13}$
El conocimiento de la etiología de estas infecciones es de suma importancia para determinar la profilaxis, así como el tratamiento empírico. En diversos protocolos se concluye que el principal microorganismo causante de infecciones en el sitio quirúrgico es Staphylococcus aureus, seguido por Staphylococcus coagulasa negativo y bacterias Gram negativas incluyendo Pseudomonas aeruginosa y Acinetobacter sp. ${ }^{13}$ Los pacientes que tienen un sistema de derivación peritoneal permanente tiene mayor riesgo de presentar infecciones por estafilococos, mientras que los pacientes con ventriculostomía presentan mayor riesgo de tener infecciones por bacilos Gram negativos. ${ }^{13}$

El empleo de profilaxis antibiótica en las craneotomías está plenamente justificado por las graves consecuencias de estas infecciones. ${ }^{14} \mathrm{El}$ uso de antibióticos profilácticos previene procesos infecciosos en aproximadamente $50 \% .{ }^{14} \mathrm{La}$ profilaxis contra estafilococo con una cefalosporina de primera generación como la cefalotina es apropiada. ${ }^{14}$ Es importante hacer mención que no se ha encontrado diferencia estadísticamente significativa con el uso de profilaxis corta (1 día aplicando primera dosis en la hora previa al inicio de la cirugía) o profilaxis extendida (7 días) para prevenir procesos infecciosos. ${ }^{15}$ Para la elección de la terapia antibiótica se debe considerar los posibles microorganismos responsables, así como la penetración de los antibióticos al sistema nervioso central. El tratamiento empírico debe incluir vancomicina más un beta-lactámico con acción contra Gram negativos. ${ }^{12}$ Debido al incremento de bacterias resistentes en este tipo de complicaciones, cabe la posibilidad de que tanto la profilaxis antibiótica, así como el tratamiento empírico utilizados puedan ser insuficientes. El tratamiento debe ajustarse según aislamientos en cultivo de líquido cefalorraquídeo y sensibilidad del microorganismo. La duración del tratamiento depende del microorganismo aislado, en el caso del aislamiento de Gram positivos se recomienda manejo por 14 
días mientras que para infecciones por microorganismos Gram negativos el tratamiento debe ser por lo menos de 21 días. $^{12}$

En los pacientes con respuesta clínica pobre, falla microbiológica o aislamiento de microorganismos resistentes a múltiples medicamentos en cultivos, además de la administración intravenosa de antibióticos, se debe considerar la aplicación intratecal o intraventricular. El beneficio de la terapia intraventricular es la presencia de concentraciones elevadas en líquido cefalorraquídeo disminuyendo el riesgo de efectos adversos sistémicos por altas dosis de medicamento parenteral para alcanzar concentraciones altas en el sistema nervioso central. ${ }^{16}$

En pacientes críticamente enfermos, atendidos en unidades de cuidados intensivos, existen varios factores de riesgo para la reactivación de citomegalovirus. La neutropenia es probablemente el factor de riesgo más frecuentemente asociado a la reactivación y mortalidad por citomegalovirus. La frecuencia se estima en $35 \%$ y en la mayoría de los casos el diagnóstico se realiza post mortem; ${ }^{17}$ sin embargo, no se ha determinado el impacto que esta reactivación tiene en el paciente críticamente enfermo, por lo que aún no conocemos el beneficio potencial de administración antiviral en estos pacientes. ${ }^{17,18}$

El paciente en discusión presentaba un mal pronóstico debido a que no fue posible resecar en la cirugía inicial más de $90 \%$ del tumor ${ }^{19}$ que, como ya se describió previamente, es el predictor más importante para la sobrevida. Además, pese al tratamiento profiláctico, al décimo día desarrolló la craniectomía ventriculitis por $P$. aeruginosa, ensombreciendo aún más el pronóstico. ${ }^{20}$ Dentro de los riesgos para desarrollar proceso infeccioso encontramos que el paciente fue sometido a dos cirugías con tiempo quirúrgico prolongado (>3 horas). ${ }^{21,22}$ Se inició tratamiento antibiótico empírico acorde a los microorganismos más frecuentemente aislados según la literatura con ceftriaxona y vancomicina para cobertura de bacterias Gram negativas y cocos Gram positivos, respectivamente, ${ }^{23}$ modificándose a meropenem por aislamiento de $P$. aeruginosa y posteriormente a cefepima por sensibilidad reportada en antibiograma. Pese al tratamiento antibiótico administrado acorde a la sensibilidad, el paciente no presentó mejoría, evolucionando a choque séptico, lo que lo llevó a su muerte.

En la necropsia, además de los datos histopatológicos de sepsis y meningoependimocoroiditis piógena, se encontraron macrófagos alveolares infectados por citomegalovirus. Esta complicación se encuentra descrita en los pacientes críticamente enfermos, en los cuales se presenta una reactivación del virus, que en raras ocasiones se sospecha y que puede ser un factor agregado para su muerte realizándose el diagnóstico habitualmente en el estudio post mortem..$^{24,25}$

\section{CONCLUSIÓN}

Los tumores cerebrales en pediatría representan la tercera neoplasia maligna más frecuente requiriendo manejo médico-quirúrgico. La resección completa es indispensable para un mejor pronóstico. Las infecciones en pacientes sometidos a craniectomía por tumor intracraneal tienen una elevada morbilidad y mortalidad incrementando el tiempo de estancia hospitalaria y costos asociados con la atención de la salud, por lo que es necesario implementar medidas de prevención incluyendo el uso de antibióticos profilácticos.

Cuando los procesos infecciosos se presentan se debe iniciar esquema antibiótico empírico a la brevedad considerando la epidemiología local, ajustando posteriormente en medida de lo posible, el esquema antibiótico según aislamiento y sensibilidad para disminuir el riesgo de muerte. En caso de falla terapéutica sistémica se debe 
considerar el uso de antibiótico intraventricular para mejorar el efecto bactericida y lograr así esterilizar el líquido cefalorraquídeo.

En pacientes críticamente enfermos se debe considerar reactivación de citomegalovirus como un factor de comorbilidad que puede llevar a la muerte a un paciente, por lo que en caso de sospecha de enfermedad, se deberá evaluar toma de serología y reacción de cadena de polimerasa para realizar un diagnóstico oportuno y evaluar a la necesidad de iniciar tratamiento con ganciclovir.

\section{REFERENCIAS}

1. Banan R., Hartmann C. The new WHO 2016 classification of briantumors-what neurosurgeons need to know. Acta Neurochir. 2017; DOI 10.1007/s00701-016-3062-3

2. Rivera-Luna R., Velasco-Hidalgo L., Zapata-Tarrés M., Cárdenas-Cardos R., Aguilar-Ortiz M. Current outlook of childhood cáncer epidemiology in a middle-income country under a public health insurance program, PediatrHematoIOncol. 2017;34:43-50.

3. Steliarova-Foucher E., Colombet M., Ries LA., Moreno F., Dolya A., et. al. International incidence of childhood cancer, 200110: a population-based registry study. LancetOncol 2017;1-13. http://dx.doi.org/10.1016/S1470-2045(17)30186-9.

4. Gupta N. et al. (eds.), Ependimoma Chapter 4, Cassie Kline, Craig Forester and Anuradha Banerjee, pp. 69-92. Pediatric CNS Tumors, Pediatric Oncology, Third Edition. Springer International Publishing 2017. DOI 10.1007/978-3-319-30789-3_4.

5. Kristian W.,Pajtler KW., Mack SC., Ramaswamy V. et. al. The current consensus on the clinical management of intracranial ependymoma and its distinct molecular variants. Acta Neuropathol. 2017;133:5-12.

6. Zhan R., Zhu Y., Shen Y., Shen J., Tong Y., Yu H., Wen L.Postoperative central nervous system infections after cranial surgery in China: incidence, causative agents, and risk factors in 1,470 patients. Eur J Clin Microbiol Infect Dis. 2014;33:861-66.

7. McClelland S., Hall WA. Postoperative Central Nervous System Infection: Incidence and Associated Factors in 2111 Neurosurgical Procedures. Clin Infect Dis. 2007;45:55-9.

8. Simon TD., Hall M., Riva-Cambrin J., et. al. Infection rates following initial cerebrospinal fluid shunt placement across pediatric hospitals in the United States.J Neurosurg Pediatr. 2009;4:156-65.

9. Kourbeti IS, Vakis AF, Ziakas P. Infections in patients undergoing craniotomy: risk factors associated with postcraniotomy meningitis. J Neurosurg. 2014;24:1-7.
10. McCutcheon BA., Ubl DS., Babu M., et. al. Predictors of Surgical Site Infection Following Craniotomy for Intracranial Neoplasms: An Analysis of Prospectively Collected Data in the ACS-NSQIP Database. World Neurosurgery. 2016;88:350-8.

11. Dashti SR., Baharvahdat H., Spetzler RF., et. al. Operative intracranial infection following craniotomy. Neurosurg Focus 2008;24:1-5.

12. Tunkel AR., Hasbun R., Bhimraj A., et. al. 2017 Infectious Diseases Society of América's Clinical Practice Guidelines for Healthcare-Associated Ventriculitis and Meningitis. Clin Infect Dis. 2017;64:e34-e65.

13. Múñez E., Ramo A., Álvarez de Espejo A., et. al. Etiología de las infecciones quirúrgicas en pacientes sometidos a craneotomía.Neurocirugia. 2012;23:54-59.

14. Alotaibi AF, Hulou MM, Vestal M, Alkholifi F, Asgarzadeh M, Cote DJ, Bi WL, Dunn IF, Mekary RA, Smith TR, The Efficacy of Antibacterial Prophylaxis against the Development of Meningitis after Craniotomy: a Metaanalysis, World Neurosurgery (2016), doi: 10.1016/ j.wneu.2016.02.048.

15. Hanak BW., Bonow RH., Harris CA.,Browd SR.Cerebrospinal Fluid Shunting Complications in Children. Pediatr Neurosurg. 2017;2:1-20.

16. Gilbert B., Morrison C. Evaluation of intraventricular colistin utilization: A case series. J Critical Care. 2017;40:161-63.

17. Arellano-Galindo J., Jiménez-Hernández E., VelásquezGuadarrama N., et. al. Frecuencia de infección activa por citomegalovirus en pacientes pediátricos en estado crítico e inmunodeprimidos. Patología. 2009;47:198-203.

18. Heininger A, Engel $C$, Notheisen $T$, Unertl $K$, Hamprecht $K$. Human cytomegalovirus infection in nonimmunosuppressed critically ill patients. Crit Care Med. 2001;29:541-7.

19. Lin FY., Chintagumpala M. Advances in Management of PediatricEpendymomas. CurrOncol Rep. 2015;17: DOI 10.1007/s11912-015-0470-0.

20. Lassen B., Helseth E., Egge A., Due-Tonnessen BJ., Ronning P., Meling TR. Surgical mortality and selected complications in 273 consecutive craniotomies for intracranial tumors in pediatric patients. Neurosurgery. 2012;70:936-43.

21. Chen $\mathrm{CH}$., Chang $\mathrm{CY}$., Lin LJ., et. al. .Risk factors associated with postcraniotomy meningitis: A retrospective study. Medicine (Baltimore) 2016;95:e4329. doi: 10.1097/ MD.0000000000004329.

22. Kurland DB., Khaladj-Ghom A., CarusilloB,. Et. al. Complications Associated with Decompressive Craniectomy: A Systematic Review.Neurocrit Care 2015;23:292-304.

23. Hasburn R. Central Nervous System Device Infections 2016 Nov;18(11):34. doi: 10.1007/s11908-016-0541-x.

24. Griffiths P1, Baraniak I, Reeves M. The pathogenesis of human cytomegalovirus. J Pathol. 2015;235(2):288-97.

25. Papazian L., Hraeiech S., Lehingue S., et. al. Cytomegalovirus reactivation in ICU patients. 2016;42:28-37. doi: 10.1007/s00134-015-4066-9. 